\title{
Lecture Notes in Computer Science
}

Commenced Publication in 1973

Founding and Former Series Editors:

Gerhard Goos, Juris Hartmanis, and Jan van Leeuwen

\section{Editorial Board}

David Hutchison

Lancaster University, Lancaster, UK

Takeo Kanade

Carnegie Mellon University, Pittsburgh, PA, USA

Josef Kittler

University of Surrey, Guildford, UK

Jon M. Kleinberg

Cornell University, Ithaca, NY, USA

Friedemann Mattern

ETH Zurich, Zurich, Switzerland

John C. Mitchell

Stanford University, Stanford, CA, USA

Moni Naor

Weizmann Institute of Science, Rehovot, Israel

C. Pandu Rangan

Indian Institute of Technology, Madras, India

Bernhard Steffen

TU Dortmund University, Dortmund, Germany

Demetri Terzopoulos

University of California, Los Angeles, CA, USA

Doug Tygar

University of California, Berkeley, CA, USA

Gerhard Weikum

Max Planck Institute for Informatics, Saarbrücken, Germany 
More information about this series at http://www.springer.com/series/7407 
Xuanhua Shi $\cdot$ Hong An

Chao Wang · Mahmut Kandemir

Hai Jin (Eds.)

\section{Network and \\ Parallel Computing}

14th IFIP WG 10.3 International Conference, NPC 2017 Hefei, China, October 20-21, 2017

Proceedings

粤 Springer 


\section{Editors}

Xuanhua Shi

Huazhong University of Science and Technology

Wuhan

China

Hong An

University of Science and Technology of China

Hefei

China

Chao Wang

University of Science and Technology of China

Hefei

China

\section{Mahmut Kandemir \\ Pennsylvania State University \\ University Park, PA \\ USA}

Hai Jin

Huazhong University of Science

and Technology

Wuhan

China

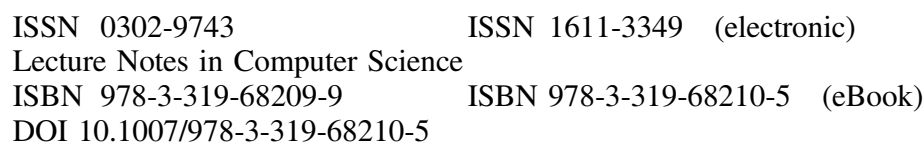

Library of Congress Control Number: 2017954908

LNCS Sublibrary: SL1 - Theoretical Computer Science and General Issues

(C) IFIP International Federation for Information Processing 2017

This work is subject to copyright. All rights are reserved by the Publisher, whether the whole or part of the material is concerned, specifically the rights of translation, reprinting, reuse of illustrations, recitation, broadcasting, reproduction on microfilms or in any other physical way, and transmission or information storage and retrieval, electronic adaptation, computer software, or by similar or dissimilar methodology now known or hereafter developed.

The use of general descriptive names, registered names, trademarks, service marks, etc. in this publication does not imply, even in the absence of a specific statement, that such names are exempt from the relevant protective laws and regulations and therefore free for general use.

The publisher, the authors and the editors are safe to assume that the advice and information in this book are believed to be true and accurate at the date of publication. Neither the publisher nor the authors or the editors give a warranty, express or implied, with respect to the material contained herein or for any errors or omissions that may have been made. The publisher remains neutral with regard to jurisdictional claims in published maps and institutional affiliations.

Printed on acid-free paper

This Springer imprint is published by Springer Nature

The registered company is Springer International Publishing AG

The registered company address is: Gewerbestrasse 11, 6330 Cham, Switzerland 


\section{Message from the NPC 2017 Chairs}

Welcome to the proceedings of the 14th IFIP International Conference on Network and Parallel Computing (NPC 2017)!

The goal of the NPC conferences is to establish an international forum for engineers and scientists to present their excellent ideas and experiences in system fields of distributed and parallel computing.

High-performance computing and big data are two main areas where NPC 2017 provided a dynamic forum to explore, discuss, and debate state-of-the-art technology issues and challenges. High-performance computers and big-data systems are tied inextricably to the broader computing ecosystem and its designs and market adoption. They also highlight information security needs and economic competitiveness in ways that distinguish them from most other scientific instruments. We strongly believe that the stakes are high, and it is far beyond the boundaries of nations and continents, and should strongly encourage a broad international participation.

For their contribution toward a successful conference, we would like to thank the reviewers for the amazing service that they provided for the NPC conference to ensure that submissions ( 88 for the conference) received the consideration and attention that they deserve. As the NPC chairs, we especially appreciate the timely completion of reviews for the final selection phases. Each paper was reviewed by three to five expert reviewers ensuring that review details were considered in the decision-making of the final acceptance of 21 full papers (slightly below 24\%), including 12 papers published as Special Issue papers of the International Journal of Parallel Programming, and nine papers published as LNCS proceedings. A number of strong papers that could not be accepted to the full papers track were considered for the short paper tracks. Finally, we selected 11 short papers (for an acceptance rate of 12.5\%). These papers cover traditional areas of network and parallel computing, including parallel applications, distributed algorithms, parallel architectures, software environments, and distributed tools.

We sincerely appreciate the work and effort of the authors in preparing their submissions for review, and addressing the reviewers' comments before submitting the camera-ready copies of their accepted papers, and attending the conference to present and discuss their work.

We also want to thank every member of the NPC 2017 Organizing Committee and Steering Committee for their help in putting together such an exciting program. Finally, we thank all the attendees.

August 2017

Xuanhua Shi

Hong An

Chao Wang

Mahmut Kandemir

Hai Jin 


\section{Organization}

\section{Organizing Committee}

General Co-chairs

Michael Gschwind

Weisong Shi

\section{Organization Chair}

Guohua Cheng

\section{Program Co-chairs}

Hong An

Mahmut Kandemir

\section{Publications Chair}

Xuanhua Shi
IBM, USA

Wayne State University, USA

Hangzhou Jianpei Technology Co., Ltd., China

University of Science and Technology of China, China

Pennsylvania State University, USA

\section{Local Arrangements Chair}

Yu Zhang

University of Science and Technology of China, China

\section{Publicity Co-chairs}

Yong Chen

Texas Tech University, USA

Guangzhong Sun

University of Science and Technology of China, China

Keiji Kimura

Waseda University, Japan

Stephane Zuckerman

University of Delaware, USA

\section{Registration Chair}

Chao Wang

University of Science and Technology of China, China

\section{Web Chair}

Hui Sun

Anhui University, China

\section{Steering Committee}

Kemal Ebcioglu (Chair)

Hai Jin (Vice Chair)

Chen Ding

Jack Dongarra

Guangrong Gao
Global Supercomputing USA

Huazhong University of Science and Technology, China University of Rochester, USA

University of Tennessee, USA

University of Delaware, USA 
Jean-Luc Gaudiot

Tony Hey

Guojie Li

Yoichi Muraoka

Viktor Prasanna

Daniel Reed

Weisong Shi

Ninghui Sun

Zhiwei Xu
University of California Irvine, USA

Science and Technology Facilities Council, UK

Institute of Computing Technology, China

Waseda University, Japan

University of Southern California, USA

University of Iowa, USA

Wayne State University, USA

Institute of Computing Technology, China

Institute of Computing Technology, China

\section{Program Committee}

Meena Arunachalam
Nachi Nachiappan
Myoungsoo Jung
Mahmut Kandemir
David Abramson
Pavan Balaji
Taisuke Boku
Sunita Chandrasekaran
Barbara Chapman
Robert Harrison
Miron Livny
Keiji Kimura
Kise Kenji
Xiaosong Ma
Yuefan Deng
Hong An
Wenguang Chen
Yeching Chung
Chen Ding
Qing Yi
Zhihui Du
Xiaobing Feng
Kai Lu
Yutong Lu
Yun Xu
Yifeng Chen
Yingwei Luo
Yong Chen
Xuanhua Shi
Weiguo Wu
Yungang Bao
Weihua Zhang
Yunquan Zhang
Li Shen

Intel, USA

Apple, USA

Yonsei University, Republic of Korea

Pennsylvania State University, USA

University of Queensland, Australia

Argonne National Laboratory, USA

University of Tsukuba, Japan

University of Delaware, USA

Stony Brook University, USA

Brook University, USA

University of Wisconsin at Madison, USA

Waseda University, Japan

Tokyo Institute of Technology, Japan

Qatar Computing Research Institute

Stony Brook University, USA

University of Science and Technology of China, China

Tsinghua University, China

National Tsinghua University, Taiwan

University of Rochester, USA

University of Colorado at Colorado Springs, USA

Tsinghua University, China

Institute of Computing Technology, CAS, China

National University of Defense Technology, China

Sun Yat-sen University, China

University of Science and Technology of China, China

Peking University, China

Peking University, China

Texas Tech University, USA

Huazhong University of Science and Technology, China

Xi'an Jiaotong University, China

Institute of Computing Technology, CAS, China

Fudan University, China

Institute of Computing Technology, CAS, China

National University of Defense Technology, China 
Chao Yang

Dongrui Fan

Chunyuan Zhang

Di $\mathrm{Wu}$

Jinlei Jiang
Institute of Software, CAS, China

Institute of Computing Technology, CAS, China

National University of Defense Technology, China

Sun Yat-sen University, China

Tsinghua University, China 


\section{Contents}

SCMKV: A Lightweight Log-Structured Key-Value Store on SCM. . . . . . . 1 Zhenjie Wang, Linpeng Huang, and Yanmin Zhu

Adaptive Length Sliding Window-Based Network Coding for Energy

Efficient Algorithm in MANETs. . . . . . . . . . . . . . .

Baolin Sun, Chao Gui, Ying Song, and Hua Chen

A Virtual Machine Migration Algorithm Based on Group Selection

in Cloud Data Center.

Zhen Guo, Wenbin Yao, and Dongbin Wang

Two-Stage Job Scheduling Model Based on Revenues and Resources . . . . . .

Yuliang Shi, Dong Liu, Jing Hu, and Jianlin Zhang

TCon: A Transparent Congestion Control Deployment Platform

for Optimizing WAN Transfers.

Yuxiang Zhang, Lin Cui, Fung Po Tso, Quanlong Guan, and Weijia Jia

Regional Congestion Mitigation in Lossless Datacenter Networks . . . . . . . . .

Xiaoli Liu, Fan Yang, Yanan Jin, Zhan Wang, Zheng Cao,

and Ninghui Sun

A Fast and Accurate Way for API Network Construction Based

on Semantic Similarity and Community Detection. . . . . . . . . . . . .

$X i$ Yang and Jian Cao

Improving Branch Prediction for Thread Migration on Multi-core Architectures . . . . . . . . . . . . . . . . . . .

Tan Zhang, Chaobing Zhou, Libo Huang, Nong Xiao, and Sheng Ma

Optimizing OpenCL Implementation of Deep Convolutional Neural

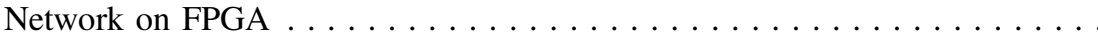

Yuran Qiao, Junzhong Shen, Dafei Huang, Qianming Yang, Mei Wen, and Chunyuan Zhang

ACO-inspired ICN Routing Scheme with Density-Based Spatial Clustering . . . . . . . . . . . . . . . . . . .

Jianhui Lv, Xingwei Wang, and Min Huang 
An Efficient Polarity Optimization Approach for Fixed Polarity

Reed-Muller Logic Circuits Based on Novel Binary Differential

Evolution Algorithm . . . . . . . . . . . . . . . . . . . .

Zhenxue He, Guangjun Qin, Limin Xiao, Fei Gu, Zhisheng Huo,

Li Ruan, Haitao Wang, Longbing Zhang, Jianbin Liu, Shaobo Liu, and Xiang Wang

Stem: A Table-Based Congestion Control Framework for Virtualized

Data Center Networks . . . . . . . . . . . . . . . . . . . . . . . . . . . . .

Jie Wu, Binzhang $F u$, and Mingyu Chen

SDAC: Porting Scientific Data to Spark RDDs . . . . . . . . . . . . . . .

Tian Yang, Kenjiro Taura, and Liu Chao

Unified Access Layer with PostgreSQL FDW for Heterogeneous Databases . . . Xuefei Wang, Ruohang Feng, Wei Dong, Xiaoqian Zhu, and Wenke Wang

Balancing Global and Local Fairness Allocation Model

in Heterogeneous Data Center. . . . . . . . . . . . . . . . . . .

Bingyu Zhou, Guangjun Qin, Limin Xiao, Zhisheng Huo, Bing Wei,

Jiulong Chang, Nan Zhou, Li Ruan, Haitao Wang, and Zipeng Wei

System Problem Detection by Mining Process Model from Console Logs . . . Jian Li and Jian Cao

Quantifying the Isolation Characteristics in Container Environments . . . . . . .

Chang Zhao, Yusen Wu, Zujie Ren, Weisong Shi, Yongjian Ren, and Jian Wan

CSAS: Cost-Based Storage Auto-Selection, a Fine Grained Storage

Selection Mechanism for Spark. . . . . . . . . . . . . . .

Bo Wang, Jie Tang, Rui Zhang, and Zhimin Gu

An In-Depth Performance Analysis of Many-Integrated Core

for Communication Efficient Heterogeneous Computing . . . . . . . . . . . .

Jie Zhang and Myoungsoo Jung

Author Index 